\title{
No explanation for the historical present: Temporal sequencing and discourse
}

\author{
Pranav ANAND - University of California, Santa Cruz \\ Maziar TOOSARVANDANI - University of California, Santa Cruz
}

\begin{abstract}
Discourses in the historical (or narrative) use of the simple present in English prohibit backshifting, though they allow forward sequencing. Unlike both reference time theories and discourse coherence theories of these temporal inferences, we propose that backshifting has a different source from narrative progression. In particular, we argue that backshifting arises through anaphora to a salient event in the preceding discourse.
\end{abstract}

Keywords: tense, discourse coherence, coherence relations, perspective.

While the present tense in English is typically described as indexical to the time of utterance, it has long been acknowledged that it can be used "historically" to describe situations that have already taken place or "narratively" for those that are simply imagined. This historical present (or, HP) is often described as more vivid or dramatic than the simple past, enabling the speaker to "recall[] or recount[] the past as vividly as if it were present" (Palmer, 1965: 39), as if the events were unfolding before her eyes (see also Leech 1971: 6-7, Close 1981: 106).

(1) Mr. Tulkinghorn takes out his papers, asks permission to place them on a golden talisman of a table at my Lady's elbow, puts on his spectacles, and begins to read by the light of a shaded lamp.

(Dickens, Bleak House)

Vividness aside, here we discuss a novel contrast between the HP and simple past that concerns how events are temporally ordered in simple multi-sentence discourses. The simple past in English evinces a famous ambiguity: its temporal interpretation can advance in tandem with linear order (2a) or be backshifted (2b).
a. $\quad$ Narrative progression
The administration fired ${ }^{e_{1}}$ Mike. He lost ${ }^{e_{2}}$ his house.
$e_{1}<e_{2}$
b. Backshifting
The administration fired ${ }^{e_{1}}$ Mike. He met ${ }^{e_{2}}$ with the ambassador. $\quad e_{1}>e_{2}$

In contrast, while the HP allows narrative progression (3a), it prohibits backshifting (3b), a fact which, to our knowledge, has not previously been observed.
a. The administration fires ${ }^{e_{1}}$ Mike. He loses $^{e_{2}}$ his house.
$e_{1}<e_{2}$
b. The administration fires ${ }^{e_{1}}$ Mike. He meets ${ }^{e_{2}}$ with the ambassador. $* e_{1}>e_{2}$

Since at least Partee (1984), the source of such temporal inferences has loomed large in the literature. Earlier reference time theories focused principally on cases of narrative progression, 
Pranav Anand and Maziar Toosarvandani

building elaborate semantic models of how the reference time of a sentence is anaphoric to preceding discourse (Partee 1984; Hinrichs 1986; Dowty 1986; Webber 1988; Kamp and Reyle 1993, a.o.). For most of these approaches, though not Webber's, reference times could never resolve to a time prior to the most recent event, and so the existence of backshifting in the interpretation of past tense discourses was a stubborn inconvenience.

More pragmatic discourse coherence theories arose in response, which posit that speakers infer coherence (or rhetorical) relations between sentences, drawing on a small inventory of such relations each with specific spatio-temporal consequences (Kehler 2002; Lascarides and Asher 1993; Asher and Lascarides 2003, a.o.). For instance, when a Narration relation is inferred between two sentences, to use Asher and Lascarides's terminology, they have a forward moving temporal interpretation; but when an Explanation (i.e., causation) relation is inferred, they instead have a backshifted interpretation.

Both these approaches assume that narrative progression and backshifting arise from the same source. Based on the contrast in $(2 \mathrm{a}-\mathrm{b})$, however, we argue that they are not a package deal. There is a distinct mechanism for backshifting that the HP explicitly interferes with. Palmer's quote above furnishes an intuitive characterization of where things go awry: the HP is a form of ersatz real-time description - backshifting is prohibited because the simulated "now" is ever moving forward. This paper advances a formal framework that makes good on this intuition.

This theory involves two independent pieces. The first is a general theory of temporal sequencing that allows narrative progression "for free," while backshifting is explicitly anaphoric (cf. Webber 1988). The second is a bicontextual semantics for present tense that unifies the canonical use of the simple present in English and the HP (Anand and Toosarvandani, to appear). As we hope to show, once the temporal sequencing system is precisely delineated, the semantics of the present tense will conspire to eliminate the possibility of backtracking.

\section{No going back!}

For discourse coherence theories, temporal sequencing arises from larger coherence effects. The contrast in $(3 \mathrm{a}-\mathrm{b})$ might, under such a view, be the manifestation of more general restrictions on intersentential discourse relations. Asher and Lascarides (2003: 471) propose something along these lines to account for the obligatory backshifting exhibited by the past perfect.

a. Max arrived ${ }^{e_{1}}$ late for work. He had taken ${ }^{e_{2}}$ the bus.

b. The council built ${ }^{e_{1}}$ the bridge. The architect had drawn up ${ }^{e_{2}}$ the plans. $e_{1}>e_{2}$

(Lascarides and Asher, 1993: 470)

Their constraint essentially eliminates all but Explanation and Elaboration discourse relations with the past perfect. In particular, it preempts Narration, which produces narrative progression.

Connections when Changing Tense (Lascarides and Asher, 1993: 471)

$\square\left(\langle\tau, \alpha, \beta\rangle \wedge s p(\alpha) \wedge p p(\beta) \rightarrow C_{p p}(\alpha, \beta)\right)$ 
Extending this idea to the contrast in $(3 \mathrm{a}-\mathrm{b})$, the HP would be prohibited from entering into an Explanation relation, which is compatible with backshifting; it would thus only occur with forward-moving relations, such as Narration. This does not stand up to closer scrutiny, however. First, an HP sentence can be coherently followed by a sentence in a range of tenses - simple past, present perfect, past perfect - that do allow backshifting interpretations. These would presumably be linked by an Explanation relation.

(6) The administration fires ${ }^{e_{1}}$ Mike. He $\{\text { met, has met, had met }\}^{e_{2}}$ with the ambassador.

$e_{1}>e_{2}$

Moreover, a sentence in the HP can be connected by the full range of discourse relations to a preceding sentence, including Explanation, just as long as there is no backshifting. In other words, two HP sentences can stand in an Explanation relation (7a), as well as an Elaboration (7b), Background (7c), or Violated Expectation (7c) relation, with the eventualities they describe simply overlapping.
a. Explanation
Liz collapses ${ }^{e_{1}}$ onto the couch. She is exhausted ${ }^{s_{2}}$ from practice. $\quad e_{1} \circ s_{2}$
b. Elaboration
Donald has $^{e_{1}}$ a lovely meal that night. He eats ${ }^{e_{2}}$ lots of well-done steak.
c. Background
Senecal opens ${ }^{e_{1}}$ the door. The room is ${ }^{s_{2}}$ pitch black. The fan is running ${ }^{s_{3}}$.
$e_{1} \circ s_{2} \circ s_{3}$
d. Violated Expectation
I offer $^{e_{1}}$ him a drink with dinner, but $\left\{\begin{array}{l}\text { he refuses } \\ \text { \#he drinks }^{e_{2}}\end{array}\right\} . \begin{aligned} & e_{1}<e_{2} \\ & e_{1}>e_{2}\end{aligned}$

In short, the restriction on intersentential discourses in the HP is a purely temporal one, not one that can be stated in terms of coherence relations

(8) Constraint on Intersentential Historical Present (CHP)

An eventuality can temporally follow or overlap the eventuality just described, but cannot temporally precede it. ${ }^{1}$

To ensure the ecological validity of the CHP, we examined N. K. Jemisin's Obelisk Gate, a recent 450 page novel written in the HP that is notable for complex temporal shifts throughout

\footnotetext{
${ }^{1}$ As we discuss in Section 5, intra-sentential relations are more liberal. Backshifting possible in the HP with overt connective (e.g., after, because, even though).

(1) a. He returns ${ }^{e_{1}}$ to the gym after he breaks ${ }^{e_{2}}$ his leg.

$e_{1}>e_{2}$

b. He cancels ${ }^{e_{1}}$ his gym membership because he breaks ${ }^{e_{2}}$ his leg.

c. Donald is forgiven ${ }^{e_{1}}$ even though he breaks ${ }^{e_{2}}$ the law.

$e_{1}>e_{2}$

$e_{1}>e_{2}$
} 
Pranav Anand and Maziar Toosarvandani

the text, often within a single chapter. The book contains many instances involving backshifting, all involving either the simple past or present perfect. A selection of cases where substituting in the simple present (for the bolded verb) yields sharp ungrammaticality follows.

(9) a. Nassun doesn't have any money beyond her allowance you see and she'd already spent that on books and sweets when word came that a lorist was in town.

b. He murmurs to her, "get your things..." Jija's mother married again a few years back and now she lives in Sume, the town in the next valley over, which will soon be destroyed utterly.

c. He's completely the same, aside from being partially turned to stone, as the days when you and he were less than lovers and more than friends. Ten years and another self ago.

d. Her teeth have been filed to points, even though sanzeds supposedly stopped doing that centuries ago.

e. There are no travelers on the road though you can tell that the ash is thinner there. People have been by recently.

f. But she tries, because once upon a time, this man was her world.

There is exactly one clear counterexample to our generalization, and it comes in a chapter that quickly sketches several crisscrossing plot threads - one at a time - across six months time. The basic skeleton of this section is delineated below.

(10) Six months pass...

Tonkee's arm survives the reattachment... She lives ${ }^{e_{1}} \ldots$

Hjarka starts courting ${ }^{e_{2}}$ Tonkee... She's mostly just confused...

Tonkee brings ${ }^{e_{3}}$ the council a new predictive model... some comm members will start showing deprivation symptoms within a year...

(p. 237)

Ykka doesn't want to tell anyone... You and the other council members agree ${ }^{e_{4}}$ reluctantly...

But because of Ykka's silence, a Breeder visits ${ }^{e_{5}}$ you a few days after you bring ${ }^{e^{\prime}}$ Tonkee home to finish recuperating.

Alabaster suffers ${ }^{e_{6}}$ another bad infection during these six months. He survives ${ }^{e_{7}}$ it only by...

$$
e_{1}<e_{1^{\prime}}<e_{5}<e_{2}<e_{3}<e_{6}<e_{7}<e_{4}
$$

The offending sentence describes a visit $\left(e_{5}\right)$ to the addressee (Essun) that happens very soon after events pick up in the chapter, but that is presented linearly rather far after the culmination of the first main thread (the prediction of a food shortage by a recently healed scientist, Tonkee).

However, this sentence itself starts another thread (about Essun's romantic entanglements), and it comes with a paragraph break that indicates the start of a new topic. Hence, it may signal a break in overall discourse coherence. Regardless of the ultimate explanation for this pattern, the 
fact that it is the only exception to our generalization in the entire novel (despite considerable temporal shifts) is a testament to its essential correctness.

\section{Problems for reference time theories}

The CHP presents significant problems for a discourse coherence theory. For reference time theories, on the other hand, it is not this generalization that is problematic, but the possibility of temporal backshifting with the simple past. The Temporal Discourse Interpretation Principle in Dowty (1986), for instance, simply prohibits it. A notable counterexample is Webber's (1988) theory, in which reference times can be anaphoric to subparts of events: anaphora to a consequent state yields narrative progression (11a), while anaphora to a prestate (preparatory phase) yields backshifting (11b).

a.

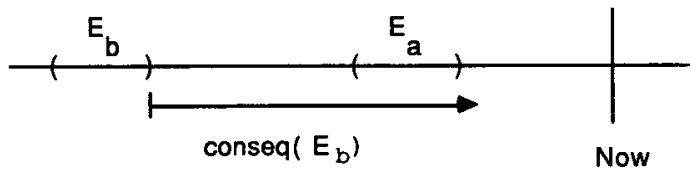

b.

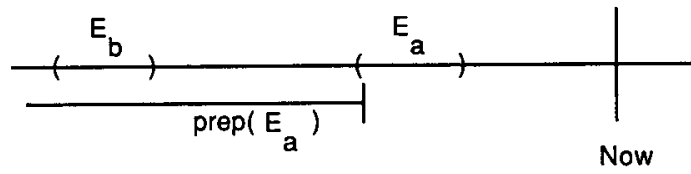

While Webber does not link these anaphoric processes to morphosyntactic categories, the CHP might be seen as evidence that they should be. The only workable move would be to stipulate that the simple present cannot be anaphoric to prestates. This derives the CHP, but it is not clear why it should be. (Note that stipulating the inverse - that HP sentences do not make their prestates available for anaphora - would not allow backshifting with the simple past, as in (6).)

There have been attempts to relate backtracking to particular aspectual combinations. For instance, Bittner (2008) argues that backshifting results from English being aspectually underspecified (unlike, say, Kallalisut). An erstwhile instantaneous event can be construed as a process, such that the event described by a subsequent sentence can be located inside the consequent state of one of its subparts. Thus, the second sentence in (2b) is backshifted when the firing event is seen as an extended process beginning at least as far back as the meeting with the ambassador. An argument for this underspecification come from what appears to be reference to an achievement as either an instantaneous event or a process. ${ }^{2}$

$$
\text { I came to the conference. }\left\{\begin{array}{l}
\text { At that instant, I knew I made a mistake. } \\
\text { The process was exhausting. }
\end{array}\right\}
$$

(Bittner, 2008: 366)

\footnotetext{
${ }^{2}$ We think there is some reason to doubt this argument. If aspectual underspecification is responsible would not easily explain why, in (2b), the two sentences can be restricted by non-overlapping temporal frame adverbials: e.g., Today, the administration fired Mike. Six months ago, he met with the ambassador. Nor for why a durative temporal adverbial, such as for the past six months, is illicit with the first sentence.
} 
Pranav Anand and Maziar Toosarvandani

However, to the extent that (12) allows both references, so does the corresponding HP version.

I come to the conference. $\left\{\begin{array}{l}\text { At that instant, I know I made a mistake. } \\ \text { The process is exhausting. }\end{array}\right\}$

Thus, if aspectual underspecification is responsible for backshifting, it should be allowed with the HP, contrary to fact.

Rather than the first sentence, it might be the backshifted sentence itself that is ambiguous. Building on Kratzer (1998), Dickey (2001) claims that the simple past in English is ambiguous between a "true" past (reference time precedes the utterance time), which leads to narrative progression, and a past perfect, which leads to backshifting. By contrast, in Dutch, Dickey argues that the simple past always yields narrative progression, citing similar facts in German. From this perspective, the CHP might arise simply because the simple present lacks this kind of morphological syncretism.

But if simple past morphology in English disguises structural past perfect, it should be possible to replicate ambiguities associated with perfects. In one such ambiguity, sentence-final temporal adverbials can constrain either the event time or the reference time; in contrast, sentence-initial adverbials only constrain the reference time (Hornstein, 1990: 24-25).

a. He had left at noon.

b. At noon, he had left.

'His leaving was at noon.'

$$
\checkmark
$$

'His leaving was at noon.'

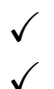

'By noon, he had already left.'

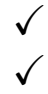

'By noon, he had already left.'

\#

\#

If the simple past permitted a past perfect structure, it should have a reference-time constraining interpretation. But this is not the case, for either a sentence-final (15a) or sentence-initial (15b) adverbial. Thus, backshifting is unlikely to derive from an ambiguity in the simple past.

\section{A bicontextual semantics for the present}

We will present a theory of temporal sequencing that treats backshifting as anaphora to the preceding discourse. This builds on a bicontextual semantics for the present tense that we have argued for elsewhere (Anand and Toosarvandani, to appear). It assumes that natural language expressions are interpreted relative to two contexts: a context of utterance $(u)$ and a context of assessment ( $a$ ). Such bicontextualism has been deployed in several empirical domains, including for free indirect discourse (Doron, 1991; Schlenker, 2004; Sharvit, 2004, 2008; Eckardt, 2015) and future tense (MacFarlane, 2003), as well as predicates of personal taste and epistemic modals (MacFarlane, 2014). 


\subsection{The semantics of tense}

Individual expression can be sensitive to one, the other, or both of these contexts. Adopting the division that Sharvit $(2004,2008)$ proposes, local pronouns are sensitive to the utterance context, while tense is sensitive to the assessment context.

$$
\begin{array}{ll}
\text { a. } & \llbracket \mathrm{I} \rrbracket^{u, a, g}=\operatorname{SPEAKER}(u) \\
\text { b. } & \llbracket \text { you } \rrbracket^{u, a, g}=\operatorname{ADDRESSEE}(u) \\
\text { a. } & \llbracket \mathrm{PRES}_{i} \rrbracket^{u, a, g} \text { is defined iff } g(i) \subseteq \operatorname{Time}(a) . \text { When defined, } \llbracket \operatorname{PRES}_{i} \rrbracket^{u, a, g}=g(i) \\
\text { b. } & \llbracket \operatorname{PAST}_{i} \rrbracket^{u, a, g} \text { is defined iff } g(i)<\operatorname{Time}(a) . \text { When defined, } \llbracket \operatorname{PAST}_{i} \rrbracket^{u, a, g}=g(i)
\end{array}
$$

Sharvit takes temporal and locatival adverbials, such as tonight (18a) and here (18b), to be sensitive to the assessment context.

$$
\begin{aligned}
& \text { a. } \llbracket \text { tonight } \rrbracket^{u, a, g}=\text { the night of the day surrounding } \operatorname{TIME}(a) \\
& \text { b. } \llbracket \text { here } \rrbracket^{u, a, g}=\operatorname{LOCATION}(a)
\end{aligned}
$$

While Sharvit assumes that the two contexts are always identical in root contexts, Anand and Toosarvandani (to appear) propose that the assessment context can be freely chosen at the root level, subject to pragmatic considerations, cf. Schlenker (2004)

$$
\begin{aligned}
& \text { Canonical Present } \\
& \operatorname{Time}(a)=\operatorname{Time}(u) \\
& \text { Historical Present } \\
& \operatorname{Time}(a)<\operatorname{Time}(u)
\end{aligned}
$$

Overlap between the assessment time and the events being described corresponds to the felt vividness of the HP, i.e., the sense of narrating something unfolding before one's eyes (Palmer 1965: 39, Leech 1971: 6-7, Close 1981: 106).

In addition, assuming that the simple present is always perfective aspect - the run time of the eventuality is contained in the reference time - only stative predicates will be compatible with the canonical present, as only they describe an eventuality small enough to fit within the assessment time, which is just as narrow as the utterance time (Cowper, 1998; Wurmbrand, 2014; Todorović, 2015). In the HP, however, the assessment time is unmoored from the utterance context, and hence it can be wide enough to contain a non-stative eventuality.

\subsection{Updating the assessment time}

The time of the assessment context can, in principle, change across sentences. After the initial segment of a discourse, we propose that is freely updated, subject only to the Constraint on Assessment Time Update (CATU). 


\section{Constraints on Assessment Time Update (CATU)}

For a sentence $S$ and an eventualities stack $E, S$ can be evaluated with respect to contexts $u$ and $a$ such that:
a. $\quad \operatorname{Time}(a):=\operatorname{TiME}(u)$, or
b. $\quad \operatorname{TimE}(a):=t$ such that $\forall t^{\prime}\left(t^{\prime}<t \rightarrow t^{\prime}<\tau\left(e_{0}\right)\right) \wedge \forall t^{\prime}\left(t^{\prime}<\tau\left(e_{0}\right) \rightarrow t^{\prime}<t\right)$

The first condition is natural: the utterance context is always a possible anchor for assessment time. The second condition invokes a stack of eventualities to which states or events are added (cf. Grosz and Sidner 1986; Webber 1988; Bittner 2003, 2005, 2008). As each sentence is interpreted, the eventuality it describes is pushed onto the stack. The top $\left(e_{0}\right)$ represents the most recent and most salient eventuality in the discourse.

$$
\begin{aligned}
& \text { Eventualities stack } \\
& E=\left\langle e_{0}, \ldots, e_{n}\right\rangle
\end{aligned}
$$

According to its second condition, CATU allows for the left boundary of $\operatorname{TimE}(a)$ and $\tau\left(e_{0}\right)$ to coincide, though there is no limit on the length of $\operatorname{TIME}(a)$. Both (23a) and (23b) are, in principle, possible updates of the assessment time. In practice, the width of the assessment time will be constrained pragmatically. The Maxim of Quantity will impose a narrower time interval, corresponding to a more informative utterance.

a.

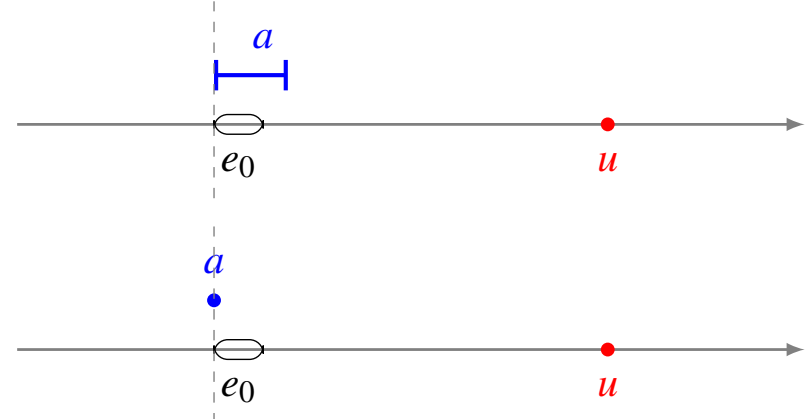

Before showing how this derives the (im)possibility of backshifting, something must be added for initial segments. At the beginning of a discourse, the assessment context can be anchored to the utterance context, but it need not be: a sentence in the HP can start off a discourse. In this case, no salient event has been introduced yet for $\operatorname{TIME}(a)$ to be updated to. So, for initial discourse segments, we propose that the assessment time can be self-anchored, so that it shares the left boundary of the the event the sentence itself describes.

\section{Constraints on Initial Assessment Times (CIAT)}

For a sentence $S$ describing an eventuality $e$ and an eventualities stack $E=\langle\rangle, S$ is evaluated with respect to contexts $u$ and $a$ such that:
a. $\quad \operatorname{Time}(a):=u$, or
b. $\quad \operatorname{Time}(a):=t$ such that $\forall t^{\prime}\left(t^{\prime}<t \rightarrow t^{\prime}<\tau(e)\right) \wedge \forall t^{\prime}\left(t^{\prime}<\tau(e) \rightarrow t^{\prime}<t\right)$. 
While CIAT determines the startup update, subsequent updates are constrained entirely by CATU. This derives the availability of backshifting with the simple past, as well as its unavailability with the HP.

\subsection{Backshifting as anaphora}

We take backshifting to arise anaphorically when $\operatorname{TIME}(a)$ is updated to left-align with the most salient event in preceding discourse - that is, the top of the event stack. For a sentence in the simple past, backshifting is thus a possibility when it follows another sentence in the simple past.

a. The administration fired ${ }^{e_{1}}$ Mike. He met ${ }^{e_{2}}$ with the ambassador. $\quad e_{1}>e_{2}$

b.

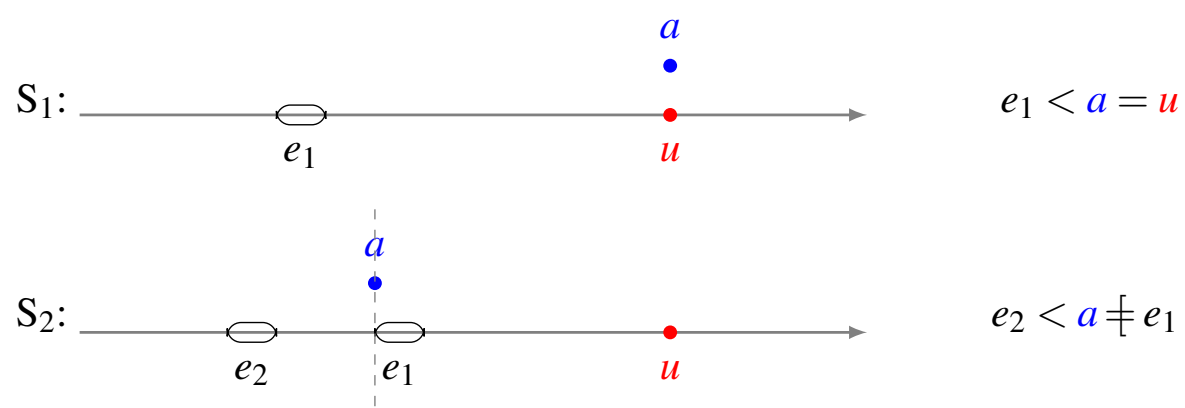

But for a sentence in the HP, backshifting is ruled out entirely. Let us start with an HP sentence following one in the simple past. Then the assessment time can be updated to left-align with the most salient preceding event, but the semantics of present tense (coupled with perfective aspect) will locate the event described inside the assessment time, rather than anterior to it.

a. The administration fired ${ }^{e_{1}}$ Mike. He meets ${ }^{e_{2}}$ with the ambassador. $* e_{1}>e_{2}$

b.

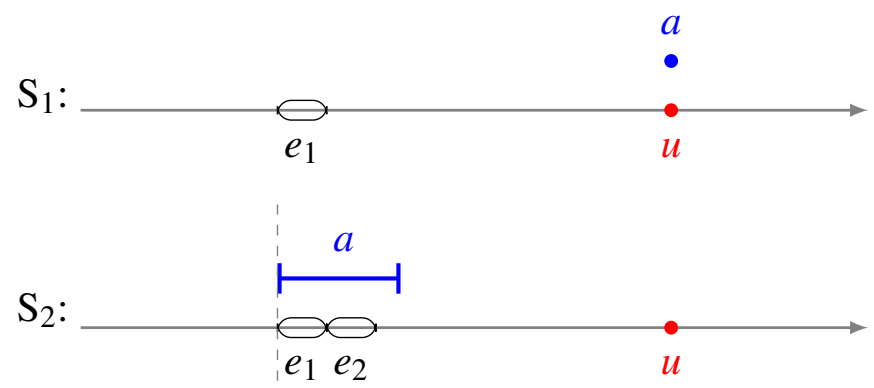

While the only interpretation the discourse in (26b) can have is a forward moving one, the earliest a sentence in the HP might be interpreted is as overlapping with the preceding sentence.

(27) The administration fired ${ }^{e_{1}}$ Mike. Trump fires ${ }^{e_{2}}$ Sean at the same time. It's ${ }^{s_{3}}$ a real bloodbath. Kellyanne is ${ }^{s_{4}}$ on TV nonstop. 
The same logic applies to a sequence of sentences entirely in the HP. The assessment time already precedes the utterance time for the first sentence. But with just the event it describes available as the top of the eventualities stack, there is no way of updating the assessment time so that the second sentence is temporally anterior, given the semantics of the present tense.

a. The administration fires ${ }^{e_{1}}$ Mike. He meets ${ }^{e_{2}}$ with the ambassador. $* e_{1}>e_{2}$

b.

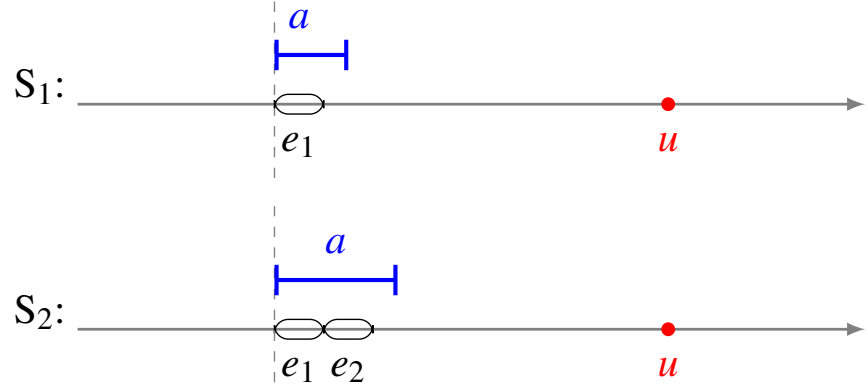

In sum, then, backshifting arises through anaphora to a salient event in the discourse. It is, as a consequence, impossible in the HP, which can only locate an event inside the assessment time.

\subsection{Backshifting with the perfect}

Just like the simple past, a sentence in the HP is able to "anchor" backshifting with a sentence in the simple past. This is also possible with the past perfect.

a. The administration fires ${ }^{e_{1}}$ Mike. $\left\{\begin{array}{l}\mathrm{He} \mathrm{met}^{e_{2}} \text { with the ambassador. } \\ \text { He had } \text { met }^{e_{2}} \text { with the ambassador. }\end{array}\right\}$

b.

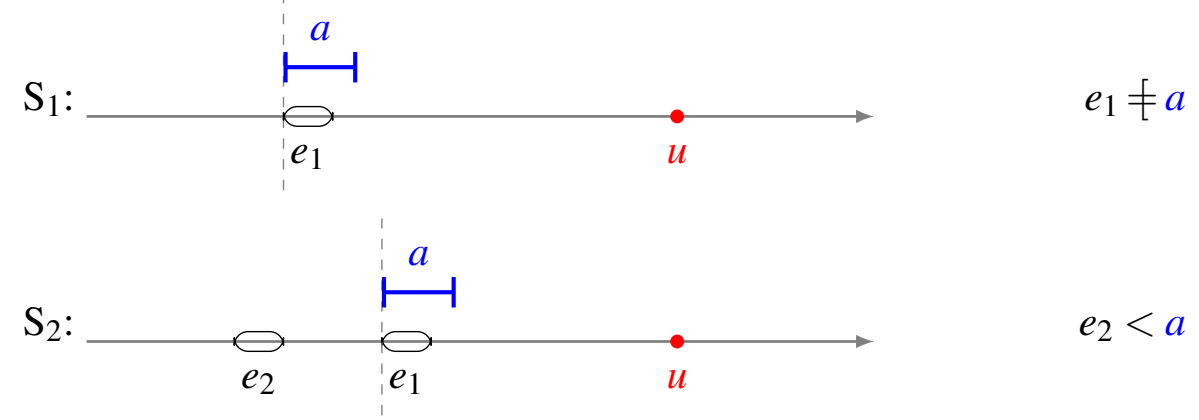

The simple past can have a backshifted interpretation here for the same reason it can in (25), except that no update of the assessment time is necessary. It is self-anchored prior to the utterance time by the first sentence, which the second sentence is then located temporally anterior to.

The equivalence of the past perfect in this discourse follows from its semantics. Building on Kamp and Reyle (1993), Anand and Toosarvandani (to appear) take it invoke a perspective 
point, which they identify with the assessment time. The past perfect would thus locate the reference time of a sentence prior to the assessment time (30b), which itself must precede the utterance (30a).
a. $\quad \operatorname{TiME}(a)<\operatorname{TIME}(u)$
b. $\quad g(i)<\operatorname{TIME}(a)$
c. $\quad \exists e(P(e) \wedge \tau(e) \subseteq g(i))$

A bicontextual semantics for the past perfect

These conditions are automatically satisfied in the discourse depicted in (29b), as the HP requires the assessment time to precede the utterance time.

Even if the first sentence is in the simple past, the past perfect will necessarily involve backshifting. To satisfy the first condition in (30), the assessment time must be updated to left-align the most salient preceding event.

a. The administration fired ${ }^{e_{1}}$ Mike. He had met ${ }^{e_{2}}$ with the ambassador.

b.

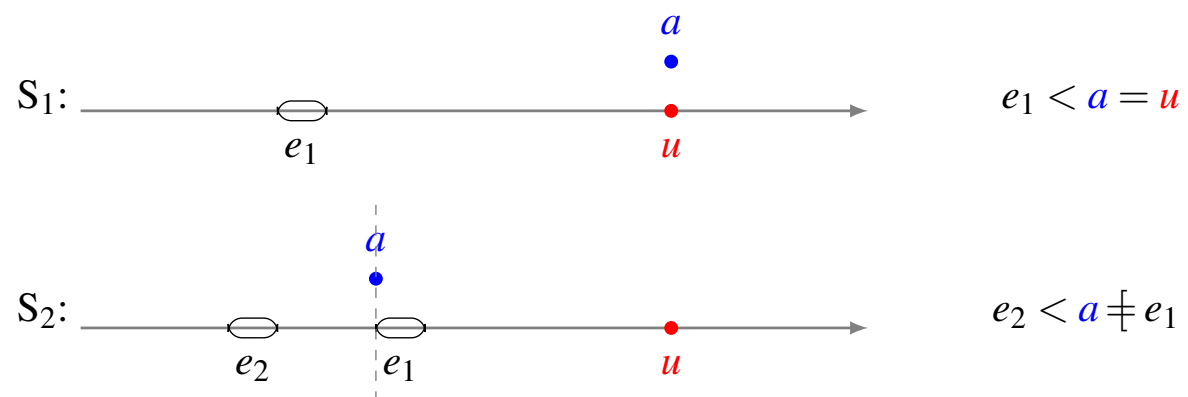

For the past perfect, then, backshifting arises as a necessary consequence of its semantics, regardless of what comes before it in the discourse.

\subsection{The present perfect}

After the HP, the present perfect, too, admits a backshifted interpretation. This is roughly equivalent to backshifting with the simple past or past perfect in (29a) above.

The administration fires ${ }^{e_{1}}$ Mike. He has met ${ }^{e_{2}}$ with the ambassador.

$$
e_{1}>e_{2}
$$

A straightforward semantics for the present perfect yields the correct result. The perfect can locate the reference time in the result state of an event, which the present tense then presupposes is included in the assessment time. 


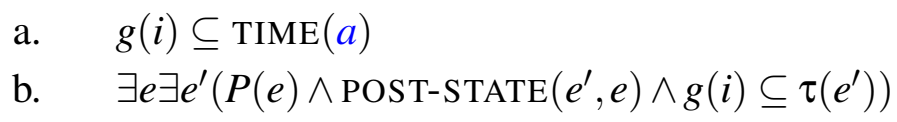

Then, just as depicted in (29b), the event described by the present perfect sentence will be anterior to the event of the first sentence.

An interesting effect arises when the antecedent sentence is in the simple past. While the present perfect is licensed with a backshifted interpretation, as in (34a), there is a felt difference to the parallel discourse with the past perfect in (31).

a. The administration fired ${ }^{e_{1}}$ Mike. He has met ${ }^{e_{2}}$ with the ambassador.

b.

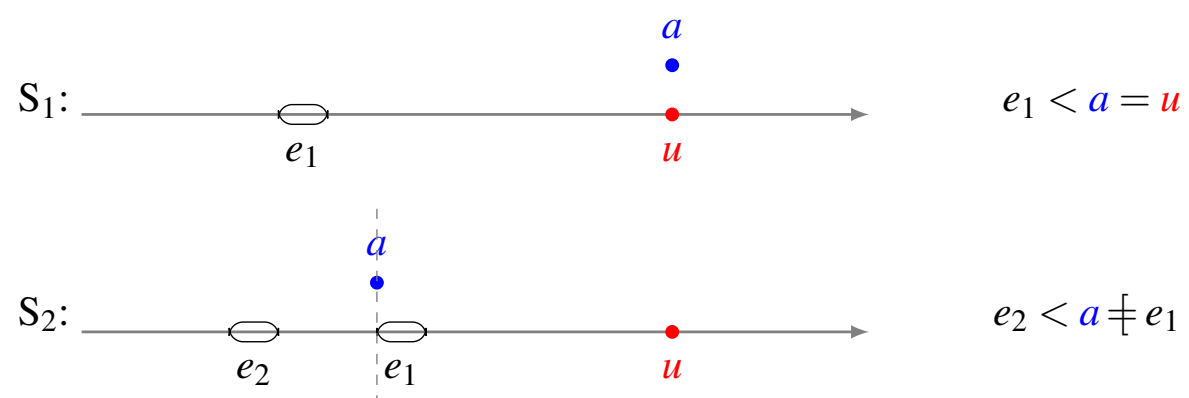

Our account permits such discourses, as the assessment time can be updated to left-align with the top of the eventuality stack for the second sentence. But it does not account for the particular effect of using the present perfect here, as opposed to the past perfect. We suspect that it may arise from other well-attested differences between the present and past perfect, including lifetime effects, current relevance, and incompatibility with temporal adverbials (see, e.g., Portner 2003).

\section{Narrative progression}

If backshifting arises through anaphora to the preceding discourse, narrative progression must derive from another mechanism that is constant across differences in tense. We will not attempt here to state directly what this mechanism is. Rather, our approach will be simply to make space for it, given our semantics for tense and the conditions on updating the assessment context.

In reference time theories, narrative progression is a direct result of temporal anaphora, as constrained by grammatical aspect (Hinrichs, 1986; Partee, 1984; Dowty, 1986; Webber, 1988). In one version of such a theory, sentences in the perfective aspect introduce a novel time interval "immediately after" the event they describe, which can serve as the antecedent for the reference time of a subsequent sentence. A sequence of simple past sentences, then, can only be interpreted as forward moving.

In discourse coherence theories, narrative progression arises from the spatio-temporal constraints associated with a specific discourse relation, such as Asher and Lascarides's (2003) Narration (see also Lascarides and Asher 1993 Altshuler 2016: 67-70), that is assumed as a de- 
fault. That is, in the absence of contradictory grammatical or other information, speakers infer that events occur in a sequence and are tightly contiguous, both temporally and spatially.

These approaches share some common intuitions. To start, they agree that narrative progression is the default, arising in the absence of information to the contrary. This is tied, in some sense, to the Maxims of Manner and Relevance, which together mandate a forward moving interpretation. And, an important constraint under both approaches is that no significant event intervene between the events in a narrative. Either one must occur "just after" another (Partee, 1984: 254) or "where things are at the end of [the first event] is where they are at the beginning of [the second event]" (Asher and Lascarides, 2003: 162). This involves considerations of Relevance.

Given these considerations, narrative progression should arise whenever it is not blocked by conflicting information - by, for instance, backshifting through anaphora to a salient event. This happens in at least two contexts. For a series of sentences entirely in the simple past, when the assessment time is not updated, nothing prevents their forward sequencing.

a. The administration fired ${ }^{e_{1}}$ Mike. He lost $^{e_{2}}$ his house.

$e_{1}<e_{2}$

b.

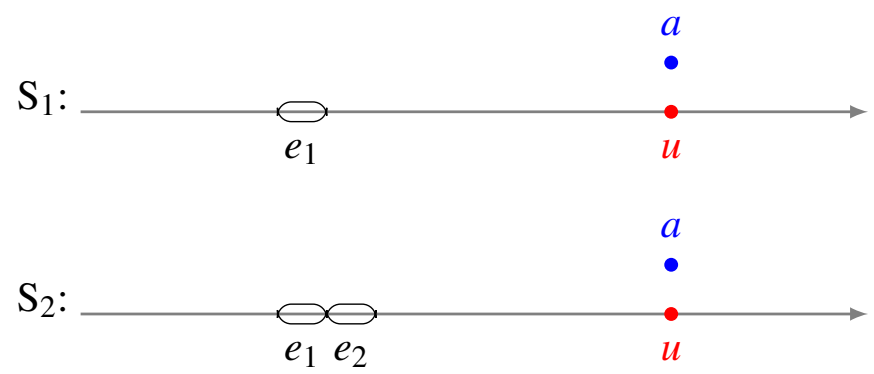

$$
e_{1}<a=u
$$$$
e_{2}<a
$$

Similarly, for a sequence entirely in the HP, each new event will be located within the same assessment time interval, which is not updated, in close temporal succession.

a. The administration fires ${ }^{e_{1}}$ Mike. He $\operatorname{loses}^{e_{2}}$ his house.

$$
\begin{array}{r}
e_{1}<e_{2} \\
e_{1} \neq a<u \\
e_{2} \subseteq a
\end{array}
$$

b.

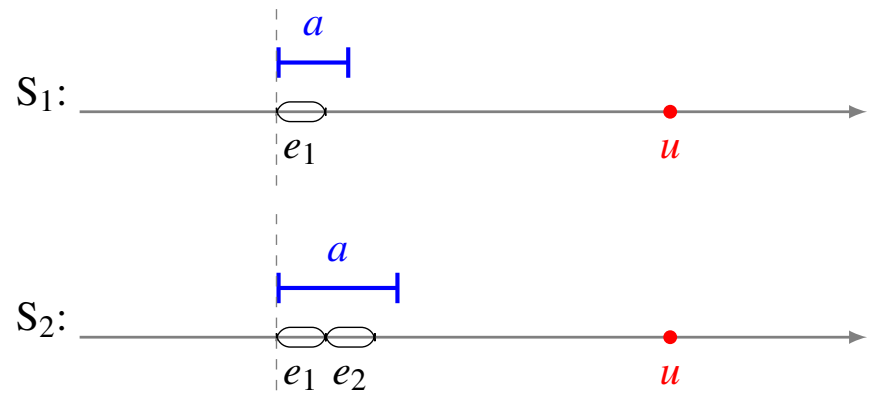

If the discourse in (36a) were extended with an additional sentence, this would necessarily describe an event located "just after" the last one, since there can be no significant events that intervene between the events already described (37a). This inference does seem like it can be 
cancelled (37b), which is compatible with it being an implicature arising from Manner and Relevance.

a. The administration fires ${ }^{e_{1}}$ Mike. He $\operatorname{loses}^{e_{2}}$ his house. \#He misses ${ }^{e_{3}}$ a house payment.

$* e_{1}<e_{3}<e_{2}$

b. The administration fires ${ }^{e_{1}}$ Mike. He $\operatorname{loses}^{e_{2}}$ his house. Actually, he first misses a house payment, and then he loses his house.

In fact, events are forward sequenced regardless of the location of the assessment time and whether it has been updated, as long as narrative progression is not prohibited. As Schiffrin (1981: 46) shows, the HP can alternate freely with the simple past without there necessarily being any backshifting.

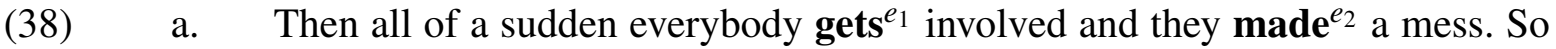
uh... this lady says ${ }^{e_{3}}$... uh this uh Bert, "Oh, my son'll make them. He's an electrician." So he makes ${ }^{e_{4}}$ them, and he charges ${ }^{e_{5}}$ all the neighbors twenty

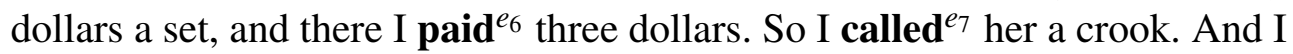
called $^{e_{8}}$ her son a crook. So, they were ${ }^{s_{9}}$ really mad at me.

$$
e_{1}<e_{2}<e_{3}<e_{4}<e_{5}<e_{6}<e_{7}<e_{8} \circ s_{9}
$$

(Schiffrin, 1981: 46)

b.

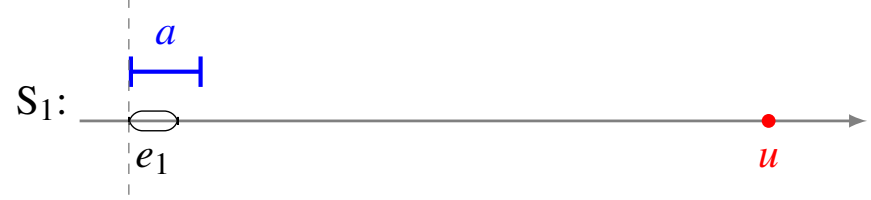

$$
a \neq e_{1}
$$
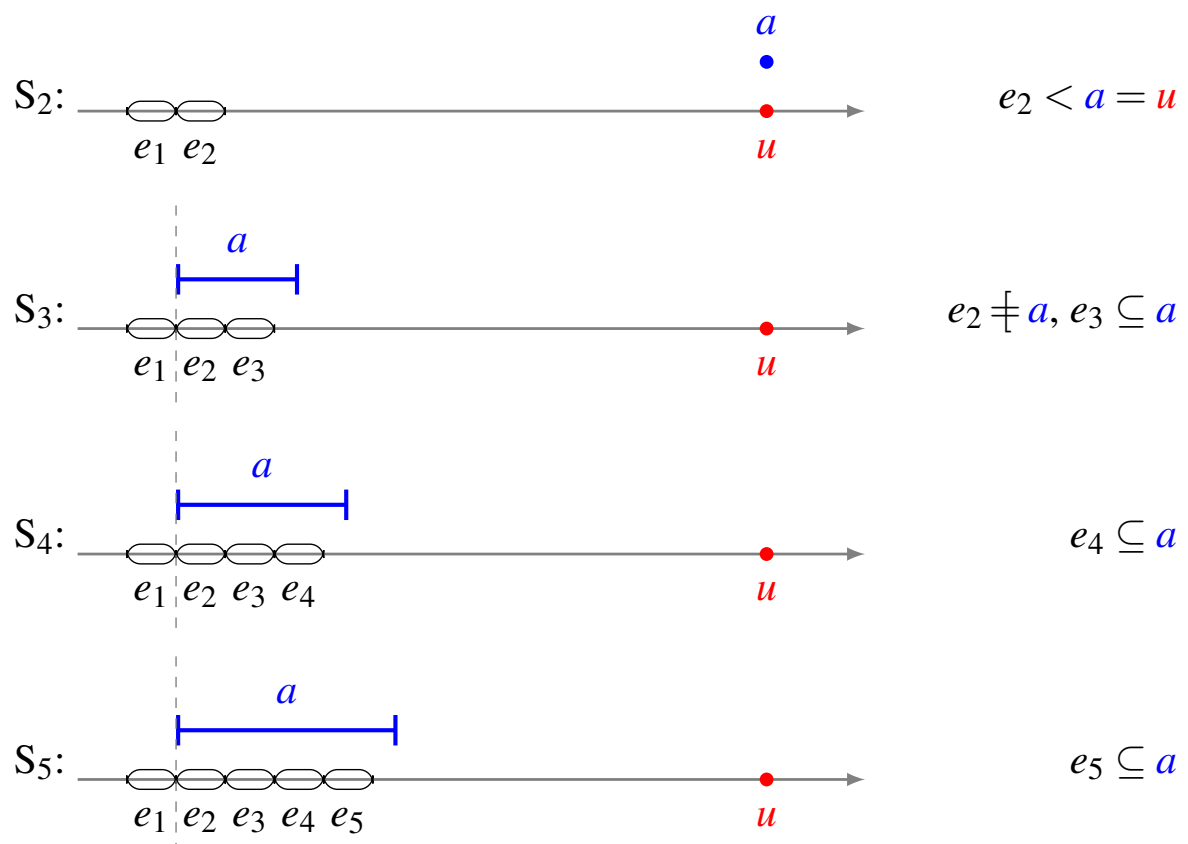

Though the assessment time is variously updated to the utterance time or the top of the event 


\begin{tabular}{lcc|lcc}
\hline \hline & FWD & BACK & & FWD & BACK \\
\hline PRES - PRES & $\checkmark(36)$ & $*(28)$ & PAST - PRES & $\checkmark(38)$ & $*(26)$ \\
PRES - PAST & $\checkmark(38)$ & $\checkmark(29)$ & PAST - PAST & $\checkmark(35)$ & $\checkmark(25)$ \\
PRES - PRES PERF & $*(32)$ & $\checkmark(32)$ & PAST - PRES PERF & $*$ & $\checkmark(34)$ \\
PRES - PAST PERF & $*(29)$ & $\checkmark(29)$ & PAST - PAST PERF & $*(31)$ & $\checkmark(31)$ \\
\hline \hline
\end{tabular}

Table 1: Summary of discourse types accounted for

stack, the principles behind narrative maintain a forward moving interpretation across the events described.

\section{Conclusion and future prospects}

Starting from the observation that backshifting is forbidden with sentences in the HP, we have motivated a more complex description of the pragmatics of temporal sequencing. At the same time, we have furnished an argument that temporal morpho-semantics can constrain discourse relations, a point that has gone unremarked given the literature's focus on past-past sequences. As Table 1 shows, our account correctly predicts interpretive possibilities for all possible continuations of simple past and HP sentences. ${ }^{3}$ Moving forward, we hope to tackle three additional, more complex discourses than considered here.

The first are intra-sentential temporal adjunct clauses, which can yield apparent backshifting in the HP.
a. He returns ${ }^{e_{1}}$ to the gym after he breaks ${ }^{e_{2}}$ his leg.
$e_{1}>e_{2}$
b. He cancels ${ }^{e_{1}}$ his gym membership because he breaks ${ }^{e_{2}}$ his leg.
$e_{1}>e_{2}$
c. Donald is forgiven ${ }^{e_{1}}$ even though he breaks ${ }^{e_{2}}$ the law.
$e_{1}>e_{2}$

Here, we suggest that, internal to a sentence, $\operatorname{TimE}(a)$ can be set wide enough to contain both events, whose order is determined entirely by the temporal connective.

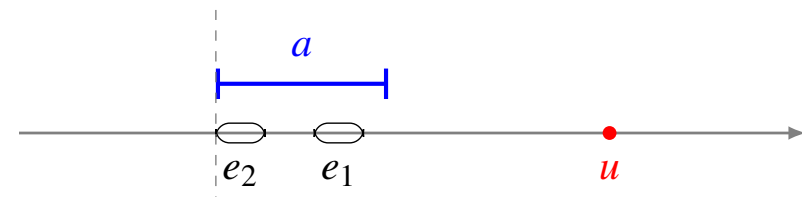

Though these adjunct clauses permit intra-sentential backshifting, they shift prior to events introduced in previous sentences. For example, (41) presents five events, with the first four

\footnotetext{
${ }^{3}$ Our system does not account for the impossibility of forward sequencing with past-present perfect combinations. However, we suspect this arises for independent reasons: the present perfect in English famously does not allow forward sequencing in general.
}

(i) Liz has entered the room. She has taken off her shoes. She has dropped her bag on a chair. 
intuitively following each other in succession. While $e_{5}$ can be understood to squeeze between $e_{3}$ and $e_{4}$, continuations which locate it before $e_{1}$ to $e_{3}$ are significantly degraded.

\begin{abstract}
Mike is nominated ${ }^{e_{1}}$ and confirmed ${ }^{e_{2}}$. Controversy swirls ${ }^{e_{3}}$. Then, the administration fires ${ }^{e_{4}}$ him because he meets ${ }^{e_{5}}$ with the ambassador (\{??before his nomination, ??before his confirmation, ??before the controversy's explosion $\}$ ).
\end{abstract}

In our current theory, two distinct principles are at play. While discourse-initially, $\operatorname{TimE}(a)$ could be constructed to contain both $e_{4}$ and $e_{5}$ in the distant past, in (41), CATU will prevent the left boundary of $\operatorname{TimE}(a)$ from retreating before $\tau\left(e_{1}\right)$ (the initial left boundary imposed by CIAT). This will then forestall $e_{5}$ from being located before $e_{1}$. For $e_{2}$ and $e_{3}$, this reasoning isn't enough, since any $e_{5}$ in between those and $e_{4}$ could still fall within the existing $\operatorname{TIME}(a)$. Can we simply insert an event between two events linked by narrative progression? What we suggested in Section 4 is that since narrative progression comes with the inference that no noteworthy or relevant event intervenes between two narratively sequenced events, the only way to do this would be to $\operatorname{shift} \operatorname{Time}(a)$ anaphorically to coincide with $e_{3}$. And given the semantics for the present tense, this would mean neither $e_{4}$ nor $e_{5}$ could precede $e_{3}$.

Beyond this issue, our theory of backshifting is currently framed in terms of a salient event, which would mean that temporal frame adverbials should not facilitate backtracking in the HP. Preliminarily, this prediction seems to hold for indexical adverbs like on Tuesday, but dependent temporal adverbials, in Hinrichs's (1986) terms, like two days earlier do seem to facilitate something akin to backshifting. We need to conduct further research into this. ${ }^{4}$

Carol is a well-liked and well-evaluated middle manager at Wells Fargo. But everything fell apart for her in the first week of January 2017.

a. On Friday, Wells Fargo fired ${ }^{e_{1}}$ her summarily. $\{$ On Tuesday, Two days earlier $\}$, she brought ${ }^{e_{2}}$ potential cases of fraud to her managers. $\quad e_{1}>e 2$

b. On Friday, Wells Fargo fires ${ }^{e_{1}}$ her summarily. \{*On Tuesday, ?Two days earlier $\}$, she brings ${ }^{e_{2}}$ potential cases of fraud to her managers. $\quad e_{1}>e_{2}$

Finally, though we have considered only two sentence discourses, it is important to consider longer and more structured discourses. It is typically understood that discourse is organized hierarchically, with backward sequencing signalling an embedded discourse segment (Grosz and Sidner 1986; Mann and Thompson 1988; Asher and Lascarides 2003, a.o.), such as the move back in (43) to a cluster of events about Fred's morning regimen.

Fred arrived ${ }^{e_{1}}$ at 10 . He had got up ${ }^{e_{2}}$ at 5 ; he had taken ${ }^{e_{3}}$ a long shower. He had got $^{e_{4}}$ dressed and eaten ${ }^{e_{5}}$ a leisurely breakfast. He had left ${ }^{e_{6}}$ the house at 6:30.

\footnotetext{
${ }^{4}$ One reason we are cautious about claiming that this is backshifting is a feeling that the two sentences are less connected; it does not feel like second sentence furnishes an explanation for the first. This is reminiscent of the feelings Dickey (2001) reports for attempted backshifting in the Dutch simple past with initial temporal adverbial topics.
} 


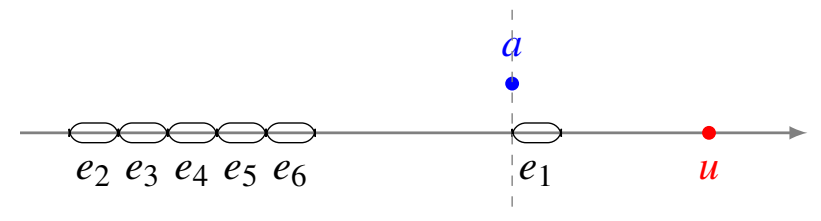

$$
e_{2}, e_{3}, e_{4}, e_{5}, e_{6}<a \neq e_{1}
$$

(Kamp and Reyle, 1993: 594)

This hierarchical organization broadens the set of possible antecedents for anaphora. While we restricted anaphoric anchors to only the eventuality described by the most recent sentence, when a discourse segment is embedded, the sentence dominating this segment also remains accessible as a possible antecedent (cf. Right Frontier Constraint; Polanyi 1988).

(44) Fred arrived ${ }^{e_{1}}$ at 10 . He had got up ${ }^{e_{2}}$ at 5. The night before, he had ironed ${ }^{e_{3}}$ his suit and had packed ${ }^{e_{4}}$ his suitcase, so he could get on the road quickly. $e_{1}>e_{2}>e_{3}<e_{4}$

a. He had left ${ }^{e_{5}}$ the house by 5:30 and gone directly to his destination. $e_{2}<e_{5}$

b. He greeted Mary as soon as he got there. $\quad e_{1}>e_{5}$

Thus, our system needs to grow to enable coherent returns to a higher discourse segment. A straightforward implementation of this would be to create stacks for each embedded discourse segment introduced. Then CATU would be understood as licensing anaphora only to those eventualities that introduce an embedded discourse segment, as only these are the top of an eventualities stack. We suspect that such an approach can handle the complex case in (10), but much further investigation is needed.

\section{References}

Altshuler, D. (2016). Events, States, and Times: An Essay on Narrative Discourse in English. Berlin: De Gruyter Mouton.

Anand, P. and M. Toosarvandani (To appear). Unifying the canonical, historical, and play-byplay present. Sinn und Bedeutung 21.

Asher, N. and A. Lascarides (2003). Logics of Conversation. Cambridge: Cambridge University Press.

Bittner, M. (2003). Word order and incremental update. Proceedings of the Chicago Linguistic Society 39(1), 634-664.

Bittner, M. (2005). Future discourse in a tenseless language. Journal of Semantics 22(4), 339-387.

Bittner, M. (2008). Aspectual universals of temporal anaphora. In S. Rothstein (Ed.), Theoretical and Crosslinguistic Approaches to the Semantics of Aspect, pp. 349-385. Amsterdam: John Benjamins.

Close, R. A. (1981). English as a Foreign Language: Its Constant Grammatical Problems (3rd ed.). London: George Allen and Unwin.

Cowper, E. (1998). The simple present tense in English: A unified treatment. Studia Linguistica 52(1), 1-18.

Dickey, M. W. (2001). The Processing of Tense: Psycholinguistic Studies on the Interpretation of Tense and Temporal Relations. Dordrecht: Kluwer. 
Doron, E. (1991). Point of view as a factor of content. Semantics and Linguistic Theory (SALT) 1, 51-64.

Dowty, D. (1986). The effects of aspectual class on the temporal structure of discourse. Linguistics and Philosophy 9, 37-61.

Eckardt, R. (2015). The Semantics of Free Indirect Discourse: How Texts Allow Us to MindRead and Eavesdrop. Leiden: Brill.

Grosz, B. J. and C. L. Sidner (1986). Attention, intentions, and the structure of discourse. Computational Linguistics 12, 175-204.

Hinrichs, E. (1986). Temporal anaphora in discourses of English. Linguistics and Philosophy 9 , 63-82.

Hornstein, N. (1990). As Time Goes By: Tense and Universal Grammar. Cambridge, MA: MIT Press.

Kamp, H. and U. Reyle (1993). From Discourse to Logic: Introduction to Modeltheoretic Semantics of Natural Language, Formal Logic, and Discourse Representation Theory. Dordrecht: Kluwer Academic Publishers.

Kehler, A. (2002). Coherence, Reference, and the Theory of Grammar. Stanford, CA: CSLI.

Kratzer, A. (1998). More structural analogies between pronouns and tense. Semantics and Linguistic Theory (SALT) 8, 92-110.

Lascarides, A. and N. Asher (1993). Temporal interpretation, discourse relations, and commonsense entailment. Linguistics and Philosophy 16, 437-493.

Leech, G. N. (1971). Meaning and the English Verb. London: Longman.

MacFarlane, J. (2003). Future contingents and relative truth. The Philosophical Quarterly 53(212), 321-336.

MacFarlane, J. (2014). Assessment sensitivity: Relative truth and its applications. Oxford University Press.

Mann, W. C. and S. A. Thompson (1988). Rhetorical Structure Theory: Towards a functional theory of text organization. Text 8(3), 243-281.

Palmer, F. R. (1965). The English Verb (2nd ed.). London: Longman.

Partee, B. H. (1984). Nominal and temporal anaphora. Linguistics and Philosophy 7, 243-286.

Polanyi, L. (1988). A formal model of the structure of discourse. Journal of Pragmatics 12(56), 601-638.

Portner, P. (2003). The (temporal) semantics and (modal) pragmatics of the perfect. Linguistics and Philosophy 26, 459-510.

Schiffrin, D. (1981). Tense variation in narrative. Language 57(1), 45-62.

Schlenker, P. (2004). Context of thought and context of utterance (a note on free indirect discourse and the historical present). Mind and Language 19(3), 279-304.

Sharvit, Y. (2004). Free indirect discourse and 'de re' pronouns. Semantics and Linguistic Theory (SALT) 14, 305-322.

Sharvit, Y. (2008). The puzzle of free indirect discourse. Linguistics and Philosophy 31, 353395.

Todorović, N. (2015). Tense and aspect (in)compatibility in Serbian matrix and subordinate clauses. Lingua 167, 82-111.

Webber, B. L. (1988). Tense as discourse anaphora. Computational Linguistics 14(2), 61-73.

Wurmbrand, S. (2014). Tense and aspect in English infinitives. Linguistic Inquiry 45(3), 403447. 